\title{
Article
}

\section{Dibromobianthryl ordering and polymerization on $\mathrm{Ag}(\mathbf{1 0 0 )}$}

Smalley, Simon, Lahti, Matti, Pussi, Katariina, Dhanak, Vinod and Smerdon, Joe

Available at https://clok.uclan.ac.uk/18707/

Smalley, Simon, Lahti, Matti, Pussi, Katariina, Dhanak, Vinod and Smerdon, Joe orcid iconORCID: 0000-0002-7387-8362 (2017) Dibromobianthryl ordering and polymerization on Ag(100). The Journal of Chemical Physics, 146 (18). ISSN 0021-9606

It is advisable to refer to the publisher's version if you intend to cite from the work. http://dx.doi.org/10.1063/1.4982939

For more information about UCLan's research in this area go to http://www.uclan.ac.uk/researchgroups/ and search for <name of research Group>.

For information about Research generally at UCLan please go to http://www.uclan.ac.uk/research/

All outputs in CLoK are protected by Intellectual Property Rights law, including Copyright law. Copyright, IPR and Moral Rights for the works on this site are retained by the individual authors and/or other copyright owners. Terms and conditions for use of this material are defined in the policies page.

\section{CLoK}

Central Lancashire online Knowledge www.clok.uclan.ac.uk

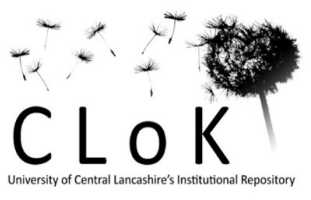




\title{
Dibromobianthryl ordering and polymerization on $\operatorname{Ag}(100)$
}

\author{
S. Smalley, ${ }^{1}$ M. Lahti, ${ }^{2}$ K. Pussi, ${ }^{2}$ V. R. Dhanak, ${ }^{3}$ and J. A. Smerdon ${ }^{1, *}$ \\ ${ }^{1}$ Jeremiah Horrocks Institute for Mathematics, Physics and Astronomy, \\ University of Central Lancashire, Preston PR1 2HE UK \\ ${ }^{2}$ Department of Mathematics and Physics, \\ Lappeenranta University of Technology, PO Box 20, FIN-53851 Finland \\ ${ }^{3}$ Stephenson Institute for Renewable Energy and Department of Physics, \\ University of Liverpool, L69 3BX, UK
}

\begin{abstract}
We study the interaction between dibromobianthryl (DBBA) and the $\operatorname{Ag}(100)$ surface using scanning tunneling microscopy and density functional theory. DBBA is prochiral on adsorption and forms racemic domains with molecular rows aligned with the substrate nearest-neighbor [011] and $[0 \overline{1} 1]$ directions. Deposition at elevated temperature leads to the formation of disordered meandering graphene nanowires of constant width.
\end{abstract}

* jsmerdon@uclan.ac.uk 


\section{INTRODUCTION}

The study of the interactions of molecules and molecular layers with surfaces has long been of interest to both fundamental and applied science. In addition to their desirable and, more importantly, customisable, electronic properties, molecules experience a range of intermolecular interactions leading to great complexity and variety in the 2- or 3-dimensional crystals that can be grown.

Recently, the rise of graphene has led to a renewed interest in depositing molecules at surfaces as a chemical feedstock for surface-mediated growth. This approach has much more promise industrially for the production of large single-layer sheets than the exfoliation of graphite [1].

A great part of the appeal of graphene is due to its potential for patterning. While continuous pristine graphene acts as an excellent conductor, electrons are confined by defects or by edges, leading to the opening of a semiconducting gap in the electronic density of states. These properties can in principle be used in digital electronic circuits formed by simply punching a particular shape out of a graphene sheet [2].

A commonly targeted shape for graphene development is the nano-ribbon (GNR), a highaspect-ratio structure a few nanometers wide and many tens or hundreds of nanometers long. A top-down approach to constructing particular architectures from graphene consists of patterning using a probe or by etching or milling, but this approach fails to give the atomic precision necessary for control over GNR edge states [3]. An alternative bottom-up route is to use molecular precursors and polymerize them into extended graphene structures.

The formation of particular structures requires precise arrangements of precursor molecules and predictable decomposition pathways. The first requirement may be met via molecular self assembly and the second via use of the Ullmann reaction, in which some extremities of the precursor that are terminated with halogen atoms rather than hydrogen atoms lose their terminal atoms at a lower temperature than others. First the structure of interest is formed via molecular self-assembly and then it is 'cured' by heating to remove halogens. A final step is heating to a higher temperature to perform cyclodehydrogenation of the structure to form continuous graphene domains.

Dibromobianthryl, (DBBA, DBMP and also referred to as dibromobianthracene and polyanthralyne in a polymerised state) shown in Figure 1(a), is composed of two anthracene 


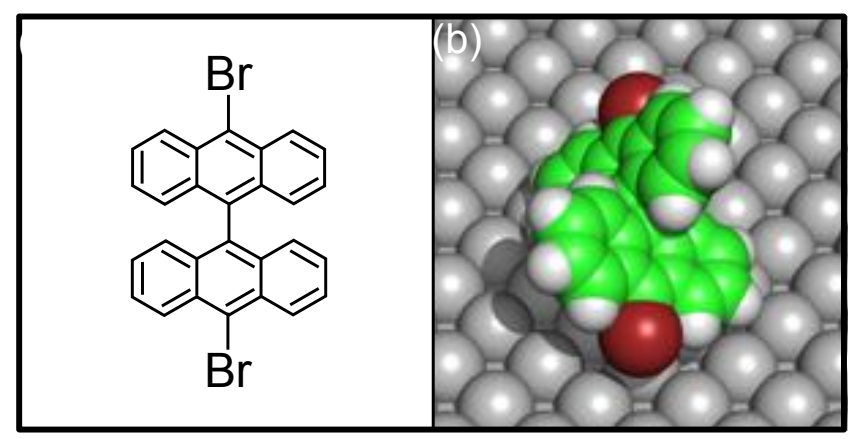

FIG. 1. (a): dibromobianthryl (DBBA), (b) one optimal configuration via DFT of isolated DBBA atop $\operatorname{Ag}(100)$.

molecules symmetrically joined in the direction across their width and, in the same direction, terminated with Br atoms [4]. One optimal configuration of DBBA atop $\operatorname{Ag}(100)$, found via density functional theory (DFT), is shown in Figure 1(b). This is further discussed in the relevant section below.

The progression of DBBA to GNRs has been described at length [5-10]. The use of DBBA has apparently been motivated by a wish to use the advantageous Ullmann dissociation reaction to polymerize DBBA in an ordered, Br-end to Br-end, fashion [5]. Following polymerization, the temperature is raised further and cyclodehydrogenation occurs, with the result that the DBBA chains flatten into GNRs as $\mathrm{C}-\mathrm{C}$ bonds form between the angled moieties. This happens as described for $\mathrm{Ag}(111)$ and $\mathrm{Au}(111)$ substrates, but, following some controversy, it has recently become unambiguously clear that the molecules line up side-by-side on a $\mathrm{Cu}(111)$ substrate, following which polymerisation into chiral GNRs occurs [10], meaning that Ullmann dissociation is not crucial for this process. This has been further supported by the successful growth of identical chiral GNRs using bianthryl precursors without terminal Br atoms, reported in the same study.

Using $\operatorname{Ag}(111)$ as a substrate, Cai et al. outlined a route to GNR formation from DBBA [5]. More recent work [8] identifies a different process to that for adsorption on $\mathrm{Cu}(111)$, with the initial debromination and cyclodehydrogenation leading to an intermediate stage of flattened graphene platelets on $\mathrm{Ag}(111)$ that can then be polymerised into GNRs through continued annealing. This behavior is markedly different from the Ullmann reaction based scheme described by Cai et al. [5].

One study reports the adsorption of DBBA atop $\mathrm{Cu}(110)$. In this case, the molecular 
film superstructure due to the underlying $\mathrm{Cu}(110)$ surface structure seems to preclude polymerization before or during cyclodehydrogenation, leading to the formation of individual platelets of graphene the size of the precursor molecules [11]. For both $\mathrm{Cu}$ surfaces, $\mathrm{Br}$ completely dissociates at $240 \mathrm{~K}$ but stays at the $\mathrm{Cu}$ surface. For $\mathrm{Cu}(110)$ dissociated $\mathrm{Br}$ atoms appear to play a role in the inhibition of graphene formation as they form an ordered network between dehydrogenated bianthryls [11]. For $\mathrm{Au}(111) \mathrm{Br}$ dissociation starts at 360

$\mathrm{K}$ and is complete at $410 \mathrm{~K}$. Desorption of Br starts with dissociation but some Br remains at the $\mathrm{Au}(111)$ surface up to complete desorption at $470 \mathrm{~K}[9]$.

No work concerning growth of GNRs has been carried out on transition metal (100) surfaces. We concentrate on this subject here, as the growth of graphene on substrates with non-hexagonal symmetry must place strain on the lattice, which may influence ribbon growth through deformation similar to that produced at conventional domain boundaries $[12,13]$. Such nanostructures have been shown to significantly alter the local electronic properties and transport characteristics [12, 14-16].

Dibromobianthryl itself is prochiral. As a free molecule, it is achiral but association with a surface restricts symmetry operations. Therefore adsorption at a surface leads to geometries which are chirally related. This is different to the helical chirality referred to in the phrase 'chiral GNRs'. The aforementioned phenomenon has no relevance to the formation of chiral GNRs as it is lost in the early dehydrogenation stage of polymerization.

Chiral assemblies and nanostructures in themselves present exciting applications in optoelectronics [17] and manifest most topically in GNR edge structures, achieved through both unzipping of carbon nanotubes [18] and in staggered or systematically offset molecular assembly [19].

\section{EXPERIMENTAL PROCEDURE}

Deposition of DBBA on to a $\mathrm{Ag}(100)$ surface was carried out via evaporation under UHV at $485 \mathrm{~K}$ using a Dodecon organic molecular beam epitaxy source. In one experiment, DBBA was deposited to submonolayer coverage on the substrate at room temperature, followed by measurements using scanning tunneling microscopy (STM). The system was then annealed to $470 \mathrm{~K}$ followed by STM. In a separate investigation, DBBA was deposited on the $\mathrm{Ag}(100)$ surface maintained at $670 \mathrm{~K}$. The $\mathrm{Ag}(100)$ surface was prepared via cycles of $\mathrm{Ar}^{+}$sputtering 
at $0.5 \mathrm{keV}$ with an ion flux of $10 \mu \mathrm{A} \mathrm{cm} \mathrm{cm}^{-2}$ for 15 minutes followed by annealing to $670 \mathrm{~K}$ for 30 minutes, measured using a Minolta LAND pyrometer with emissivity set to 0.1. Prior to deposition the DBBA source was outgassed at $490 \mathrm{~K}$. Source-sample separation during deposition was $\sim 10 \mathrm{~cm}$ and the deposition duration was $180 \mathrm{~s}$ for all experiments. STM was performed on an Omicron RT-STM1 equipped with the MATRIX control system. The scanner was calibrated with a single calibration coefficient measured at $10 \mathrm{~nm}$ scan range using the clean $\operatorname{Ag}(100)$ surface. The tips used were commercially manufactured W tips.

\section{COMPUTATIONAL DETAILS}

The static calculations for total energies were performed using the Vienna ab initio simulation package (VASP) [20-23] including the projector augmented wave (PAW) [24] potentials. A kinetic energy cut-off of $400 \mathrm{eV}$ was applied for the plane waves. The exchange and correlation functionals were treated by the generalized-gradient approximation (GGA) of Perdew-Burke-Ernzerhof (PBE) as proposed by Perdew, Burke, and Ernzerhof [25]. The $6 \times 6 \times 1$ Monkhorst-Pack mesh [26] was used for $k$-point sampling. The Ag surface was mod-

elled using the supercell approach, where periodic boundary conditions are applied to the central supercell so that it is reproduced periodically throughout $x y z$-space. The surface slab was modelled with 5 layers of Ag atoms. A region of approximately $20 \AA$ of vacuum was inserted in the $z$-direction to prevent interactions occurring between periodic images. The bottommost layer of the surface slab was frozen during the geometry relaxation. For the description of long-range van der Waals (vdW) energy the optB86b-vdW functional [27] was used.

The lattice constant that DFT gives depends on the functional used. Standard PBE overestimates bulk lattice constants due to the inherent tendency of the local-density approximation (LDA) to distribute charge uniformly which is not the case, especially in metals. The vdW density functional (vdW-DF) method better predicts the lattice constant through the formulation of the long-range dispersive effects on the correlation part and the optimization of exchange energy, so called the optB86b functional. In our calculation the lattice constant value is $4.10 \AA$, which is close to the experimental value of $4.07 \AA$. 


\section{RESULTS AND DISCUSSION}

\section{A. DBBA adsorption at room temperature}

Submonolayer DBBA on the $\operatorname{Ag}(100)$ surface at room temperature forms islands composed of ordered molecular domains, shown in Figure 2. As these appear in two orientations at approximately right angles, we conclude that they are aligned with high-symmetry axes in the $\mathrm{Ag}(100)$ surface. These may be either the [010] and [001] crystallographic primary axes or the [011] and [011] nearest-neighbor axes. Any other relationship with surface axes would lead to degenerate ordering and more observed azimuthal domain orientations. We explore this argument in more detail later. Orientation parallel to the nearest-neighbor axes ([011] and [011]) fits better with the atomic resolution data shown in Figure 1(a).

Significantly different scanning parameters are required to image the molecules and the substrate with atomic resolution, so the two do not coexist in the same frame. This is an oft-encountered issue with determining molecular ordering at surfaces. We therefore present atomic resolution data collected within the same experimental run in the inset to Figure 2 (a). In Figure S2 in Supporting Information (SI) we present data from a new sample and new orientation, leading to two different sets of primary axes for the two runs and showing the same alignment.

The coexistence of both island orientations in Figure 2(b) allows the absolute correction of thermal drift. We distort the image to give the image in the lower portion of Figure 2(b), in which the rows in differing domains are perpendicular to one another with a matching equivalent molecular spacing within each row.

In our dataset, covering approximately 2 microns on 2 different samples, we see only 2 island orientations per sample. Given a single molecule which tends to agglomerate to form islands, adsorption on a four-fold substrate leads to two possibilities: either, the molecule experiences no interaction potential energy surface corrugation and forms azimuthally disordered islands, or does experience corrugation thereof, hence forming azimuthally ordered islands. If the molecule is perfectly 'square' it is likely that it will form islands in a single orientation. If the molecule is 2-fold, such as DBBA, the minimum number of island orientations is 2, with the 2-fold axis coincident along either one (nearest-neighbor (NN), [011], $[0 \overline{11}])$ or the other $(\sqrt{2} \cdot \mathrm{NN},[010],[001])$ group of substrate high symmetry axes. More than 


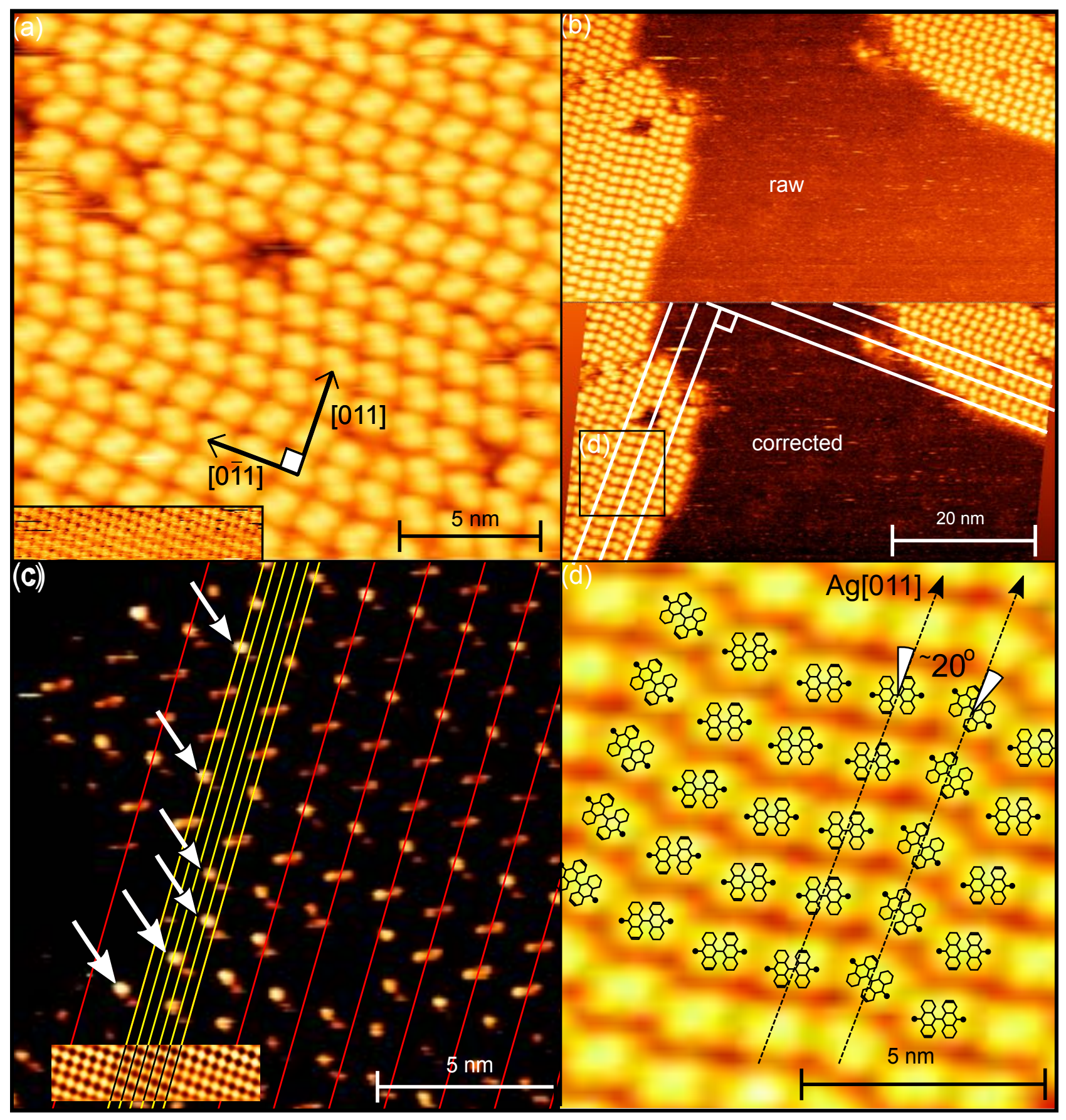

FIG. 2. (a) Scanning tunneling topograph of a single racemic domain of DBBA $/ \operatorname{Ag}(100)\left(V_{B}=\right.$ $\left.2.5 \mathrm{~V}, I_{T}=100 \mathrm{pA}\right)$ Inset: Atomic resolution on the clean $\operatorname{Ag}(100)$ surface $\left(V_{B}=-1 \mathrm{~V}, I_{T}=\right.$ $1.5 \mathrm{nA}$ ), (b) Raw and corrected STM topographs of simultaneous resolution of two racemic domains at right-angles $\left(V_{B}=-2.5 \mathrm{~V}, I_{T}=100 \mathrm{pA}\right)$. The corrections applied are plane-correction and $x$ and $y$ scale and skew factors inferred from the rotated but otherwise equivalent domains. The data for panel (d) is indicated. (c) Contrast enhanced STM topograph. Red lines indicate intra-row molecular separation. Yellow lines indicate surface atomic separation for $\mathrm{p}(5 \times 5)$ superstructure. White arrows indicate molecules of the same chirality. In this image, tip LDOS asymmetry leads to a difference in contrast for the two chiralities. Inset Fourier filtered image of the $\mathrm{Ag}(100)$ surface plotted at the same scale as the image. (d) The proposed approximate orientations for the DBBA molecules superimposed on the detail indicated ${ }^{7}$ in $(b)$. 


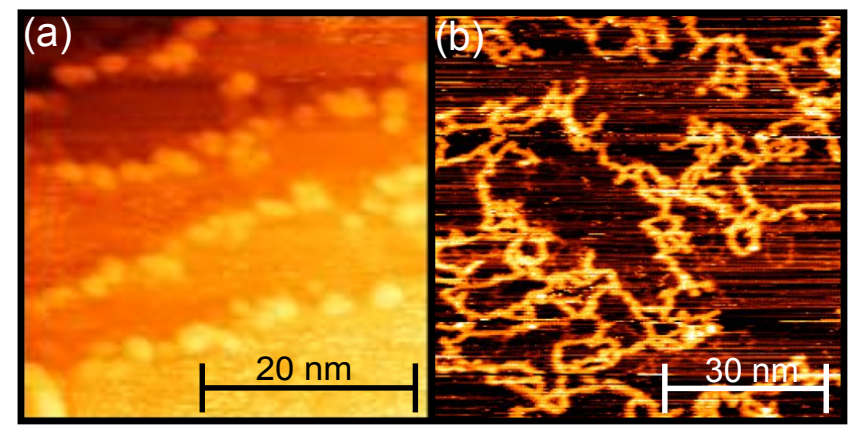

FIG. 3. (a) Scanning tunneling topograph taken after annealing to $470 \mathrm{~K}\left(V_{B}=-3.5 \mathrm{~V}, I_{T}=\right.$ $50 \mathrm{pA})$. (b) Disordered graphene nanoribbons formed during deposition of DBBA on $\mathrm{Ag}(100)$ heated to $670 \mathrm{~K}\left(V_{B}=-2.5 \mathrm{~V}, I_{T}=100 \mathrm{pA}\right)$.

2 arrangements are possible: any deviation from alignment along a high symmetry axis must then have the high-symmetry axis as a degenerate mirror axis, as these adsorption situations are necessarily energetically equivalent. The 2 orientations we see must therefore be symmetrically equivalent with the only difference being alignment along one or the other substrate high symmetry direction. Therefore, the angle between the islands must be $90^{\circ}$ and the intermolecular separation in the rows must be equal.

From the corrected data we determine the overlayer superstructure. First, we note that the unit cell is square. This is clear from the drift-corrected data: the inter-row separation of one domain determines the intra-row separation of the right-angled domain, and vice versa. The staggering between domains must be quantized by the $\mathrm{Ag}(100)$ atomic separation, which we find to be one-fifth of the molecular unit cell, shown in Figure 2(c). We ensure that only molecules of the same chirality are considered by choosing an STM image in which asymmetry in the tip local density of states (LDOS) leads to a different appearance for different DBBA chiralities. This leads to the conclusion that the DBBA structure is described primarily by a $\mathrm{p}(5 \times 5)$ arrangement.

Applying this description to corrected data of a single domain, at a shorter length scale, gives a clearer picture for determining molecular alignment with respect to the substrate as shown in Figure $2(\mathrm{~d})$. The central axes of the molecules seem to lie at approximately $20^{\circ}$ to the domain axes (and therefore the substrate $\mathrm{NN}$ axes).

There are 2 types of molecular row in the domains which appear to be oriented differently to each other. The two remaining degrees of freedom available to a DBBA molecule of fixed 
location and molecular plane are azimuthal orientation and chirality. For a (100) surface, the azimuthal orientation is quantized to $(90 \pm \theta)^{\circ}$, where $\theta$ is some degenerate domain angle. As argued above, the 2 domains observed in our data dictate that $\theta=0^{\circ}$. Chirality is expressed upon adsorption as either top left corner up or top left corner down, which is sufficient to determine the positions of the other three corners of the molecule.

We find that within a given domain, the freedom expressed is chirality; i.e., different molecules within a given domain have mirror symmetry about the substrate NN axes. DBBA is prochiral and 2-fold rotationally symmetrical about the center azimuth, so the two different chiralities are completely superimposable except due to the constraint by a surface. Therefore the interactions each chirality has with the surface are completely symmetrically equivalent and identical in every way except that they are not superposable.

As the chirality of each row is random, each domain island is approximately racemic. This contrasts with the situation where prochiral molecules adopt an inherently racemic structure with a unit cell including one of each enantiomer, as observed for e.g. dicarboxystilbene on $\mathrm{Cu}(111)$ [28]. For the opposite domain, the mirror symmetry of inequivalent molecules is maintained and the molecules are at right-angles to those in the first domain.

As mentioned earlier, the chirality referred to here is unrelated to the edge chirality of GNRs grown on $\mathrm{Cu}(111)$. It is due to the prochirality of DBBA in the shape of the relative rotation of the anthryl moieties and is manifested when adsorbed on a surface.

In studies using $\mathrm{Cu}(110)$ and (111), Br dissociates from the molecules well below room temperature. However, these atoms do not desorb completely until $620 \mathrm{~K}$ [9], and remain as distinct spherical features that stay at the periphery of assemblies of bianthryls, polyanthryls or GNRs [11] - that is, Br atoms are visible as spherical features throughout several stages in the formation of GNRs. The spherical features also seem to appear in STM data from DBBA/Ag(111) after heating to $450 \mathrm{~K}$, but an explanation thereof is not given in the article [8]. We do not see these features at any point, indicating that the $\mathrm{Br}$ is still attached to the DBBA molecules at room temperature. Following annealing to $470 \mathrm{~K}, \mathrm{Br}$ has desorbed completely from $\mathrm{Ag}(100)$, as detected via x-ray photoelectron spectroscopy data (shown in $\mathrm{SI})$.

The observation of just four situations for molecules, in keeping with the 4-fold symmetry of the surface and the prochirality of the molecule indicates that the two rotations and chiral conformations are geometrically identical in terms of their relationship with the surface. 
Rotational symmetry breaking is limited to the scale of the maximum domain size, which, while larger than any island we observe, is still on the sub-micron scale, as determined by the domain separation. In addition it only runs along rows; as mentioned above, each row has a random chirality.

Following dehalogenation of the DBBA by raising the temperature to $470 \mathrm{~K}$, ordered molecular material is not observed via STM. We observe disordered clumps at step edges surrounded by noise, as in Figure 3(a). Cyclodehydrogenation of dehalogenated DBBA (that occurs prior to polymerization) has been observed on $\mathrm{Ag}(111)$ at temperatures of $450 \mathrm{~K}$ [8]. Hence, it seem likely that the disordered material we find at step edges is agglomerations of these nanoplatelets.

\section{DBBA adsorption at elevated temperature}

For adsorption of DBBA on $\mathrm{Ag}(100)$ maintained at $670 \mathrm{~K}$, we observe disordered linear features, as reproduced in Figure 3(b). These indicate that polymerisation does occur under these adsorption conditions. However, in contrast to the behavior for the $\mathrm{Ag}(111)$ surface, sustained linear growth appears less favored on the (100) surface as seen in Figure 3(b). We do not observe the 'zig-zag' pattern associated with polymerized DBBA. The linear features are essentially flat. This is expected if the DBBA dehydrogenates to form nanoplatelets prior to polymerisation. The width of GNRs seen in our data of $\sim 1 \mathrm{~nm}$ seems consistent with single DBBA chains of 7 carbon rows. GNRs with greater widths are evident but appear sporadically, with a tendency to sit at chain convergence points (Figure 3(b)). GNRs wider than $1 \mathrm{~nm}$ never extend beyond $10 \mathrm{~nm}$ in length for the low coverage observed. In addition to this we also see brighter regions present in the GNRs. These are prevalent mostly at wider regions and junctions within the GNR. This appearance may arise from lattice deformations at GNR junctions leading to altered topography or LDOS. The same has been observed at GNR terminations on $\mathrm{Ag}(111)$ and $\mathrm{Au}(111)$ [29].

The lack of extended 1-dimensional growth points to the existence of more coupling arrangements than the end-to-end Ullmann reaction. In fact, it may be that the Ullmann reaction is not important in this system, as has been shown for DBBA adsorption atop $\mathrm{Cu}(111)[10]$. 


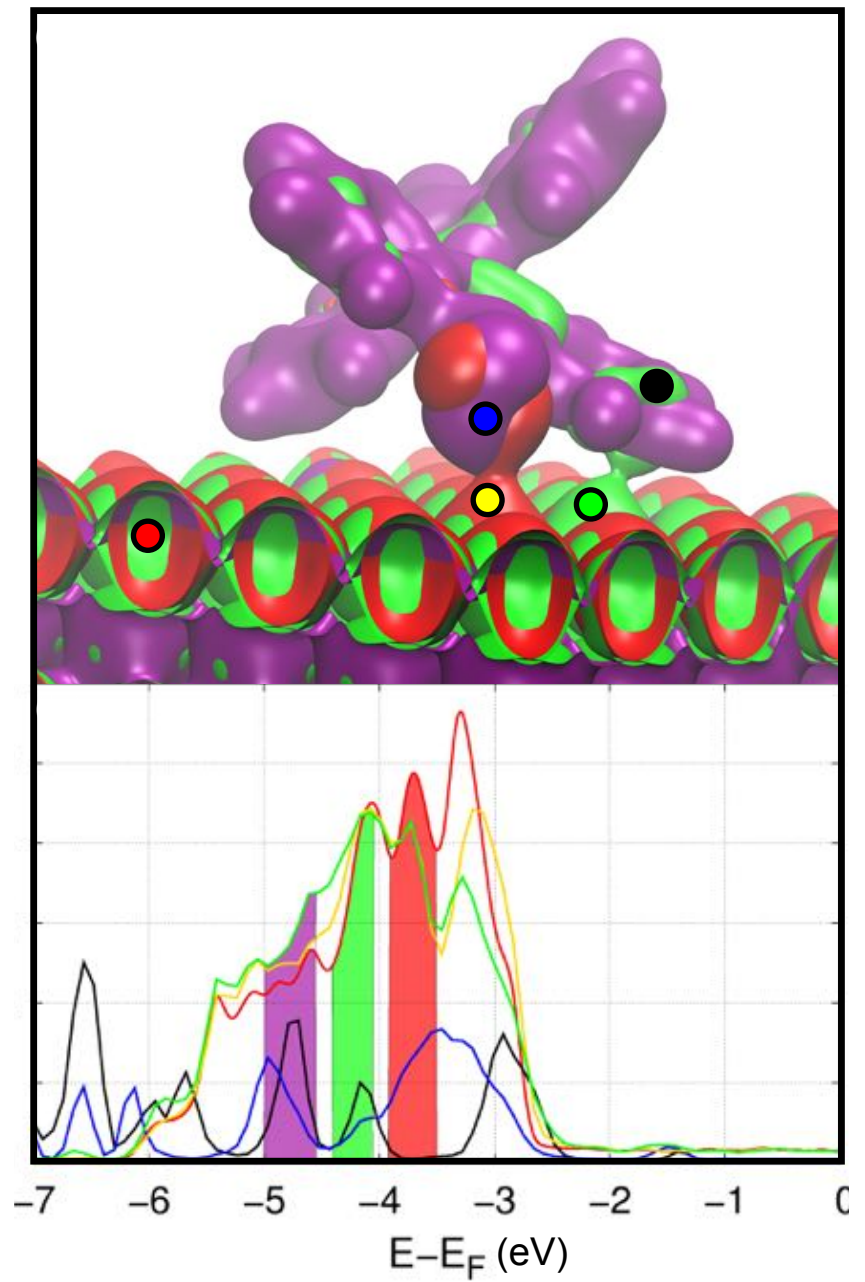

FIG. 4. (a) Partial charge density map of a DBBA molecule on $\operatorname{Ag}(100)$. The energy ranges of the colored zones are indicated in the density of states curves shown in (b). The curves are for the atoms indicated with the dots shown on the map.

\section{DFT}

First, we use DFT to find the adsorption site for a single molecule, shown in figures 1(b) and 4(a). Coordinates for the relaxed model are given in SI. We find that many azimuthally related sites exist with approximately the same adsorption energy. The main feature of the adsorption is that sites are preferred which reduce the minimum $\mathrm{C}-\mathrm{Ag}(100)$ distance. This appears to principally support adsorption even if only one $\mathrm{C}$ atom can get closer than others. In general, adsorption energies are low, in the region of $1.7-1.8 \mathrm{eV}$ per molecule. We attribute this to the crossed three-dimensional shape of the molecule preventing a close 
association between surface electrons and the $\pi$ systems of the anthryl groups. Bader analysis shows that there is no charge transfer from the substrate to the DBBA.

The bonding of the DBBA molecule (shown in Figure 4) is far from simple regardless of the apparent contact between the molecule and substrate. Different energy ranges for bonding can be identified: the red region represents $\mathrm{Br}$ atom bonding, the green region $\mathrm{C}$ atom bonding and in the purple region bonding is seen for both $\mathrm{Br}$ and $\mathrm{C}$.

DFT finds that adsorption is preferred $(-0.3 \mathrm{eV} /$ molecule $)$ with one $\mathrm{C}$ end and so one $\mathrm{Br}$ atom closer to the surface than the other. This is not detectable in STM topographs. The magnitude of the tilt is such that it ought to be visible, so we conclude that at room temperature, the molecules are able to oscillate between degenerate tilted states. On $\operatorname{Ag}(111)$, the tilt seems to be visible (in SI of reference [8]). The closer surface Ag packing suggests a tighter DBBA packing that may lock in the tilt on the (111) surface.

DFT does not show spontaneous debromination. In fact, when dissociated $\mathrm{Br}$ and debrominated DBBA are coadsorbed, they reattach during the simulation. Thus debromination requires some external energy. This is in keeping with our results that do not show evidence of debromination at room temperature.

\section{A. Azimuthal orientation of individual molecules}

There has been some controversy regarding the formation of purely armchair versus partially chiral GNRs from DBBA on $\mathrm{Cu}(111)[9,10]$. This originated in differing interpretations of the relative orientation of DBBA molecules in the rows of molecules that form as precursors to GNRs. The options for relative orientations are shown in Figure 5.

The driving force behind one orientation is interaction between $\mathrm{Br}$ atoms on adjacent molecules. For the other it is interaction between $\mathrm{H}$ atoms. The former leads to achiral GNRs and the latter to chiral GNRs. Although for $\mathrm{Cu}(111)$ it was unambiguously shown that the second arrangement is in fact the correct one - and additionally, Br plays no role in the polymerization process - the first arrangement has been indirectly observed on $\operatorname{Ag}(111)$ [8] and $\mathrm{Au}(111)$ [5] as the GNRs formed are achiral.

DFT shows negligible preference for any particular azimuthal orientation at $0 \mathrm{~K}$. We

find that there is very little effect on the adsorption energy when azimuthally rotating the molecule in the unit cell of a domain $(\sim 0.1 \mathrm{eV})$. This indicates that the orientation preference 


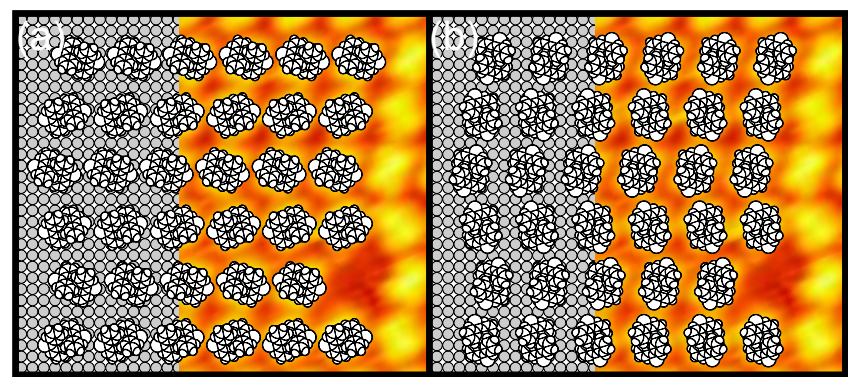

FIG. 5. The two options for azimuthal orientation in the $\mathrm{p}(5 \times 5)$ superstructure, depending on the intermolecular interaction. (a) oriented with $\mathrm{Br}-\mathrm{Br}$ axes at $\pm 20^{\circ}$ to the molecular row axis. (b) oriented with $\mathrm{Br}-\mathrm{Br}$ axes perpendicular to lines at $\pm 20^{\circ}$ to the molecular row axis. The figure is to scale with the portions of STM topography shown. Further discussion in text.

originates in intermolecular interactions rather than influence from the substrate. The size of the unit cell does affect the energy of adsorption; the best unit cell by DFT is $\mathrm{p}(4 \times 4)$. As we observe $\mathrm{p}(5 \times 5)$ superstructure via STM, we are led to the conclusion that that room temperature thermal excitations are allowing the molecule to move between all the available local minima in the adsorption potential energy surface, which increases their apparent size, forcing the $\mathrm{p}(5 \times 5)$ structure and also forcing intermolecular interactions that stabilise one orientation for all molecules in a row. When the molecules are in a p $(5 \times 5)$ structure (or larger), we find no orientation-dependent intermolecular interaction strong enough to affect the adsorption energy to within 3 significant figures.

As shown in Figures 2(d) and 5, the topography of DBBA molecules from STM is measured as square, with diagonally opposite corners bright (or dark). This is a difficult shape to unambiguously assign an orientation to. The most likely options, considering the scanning tunneling topographs, are shown in Figure 5. Panel (b) has similar orientation to that found for $\mathrm{Cu}(111)$. The molecular $\mathrm{NN}$ is $1.4(4) \mathrm{nm}$, which is consistent with the molecular domains measured atop $\mathrm{Cu}(111)$ and $\mathrm{Cu}(110)$, though comparisons are limited as debromination is spontaneous at RT on these substrates.

The best adsorption site by DFT is not symmetrical. As argued above, since we only observe 4 kinds of molecules in this film, the molecules must have thermal motion and have time-averaged occupation of all the sites with adsorption energies below their thermal energy. This would increase their interaction range slightly and also give them the symmetry observed. 
However these observations cannot help in unambiguously determining the azimuthal orientation of the molecules within the $\mathrm{p}(4 \times 4)$ superstructure. By inspection, it would appear that the STM images most closely match that shown in Figure 5(b), which would mirror the interaction found on $\mathrm{Cu}(111)$ [10].

\section{CONCLUSIONS}

We have deposited dibromobianthryl onto the $\mathrm{Ag}(100)$ surface at room temperature. We find that the DBBA molecule shape leads to prochiral adsorption. The molecules agglomerate into domain islands composed of rows of end to end molecules. Although the domains have a predominantly square $\mathrm{p}(5 \times 5)$ superstructure, adjacent rows are sometimes staggered with registration quantized to the Ag NN distance. Rather than the azimuthal rotation defining different rows within a domain, we find instead mirror-flipped molecules defining different rows within domains that are on average racemic.

Following the four-fold symmetry of the substrate, racemic right-angle rotated domains also exist, which again consist of randomly staggered rows related by chiral, rather than azimuthal, symmetry.

Moderate heating causes the loss of Br from the molecules and surface. The molecules polymerise into clumps and randomly decorate the step edges.

We have also deposited DBBA directly on a $\mathrm{Ag}(100)$ surface maintained at high temperature. The molecules polymerise into chains that are short and meandering, indicating that it is unlikely that end-to-end Ullmann coupling is the sole polymerisation mechanism.

DFT analysis indicates that $\mathrm{C}$ dominates the molecule-surface interaction and that spontaneous dehalogenation requires some external energy on $\operatorname{Ag}(100)$. There is very poor selectivity of adsorption site at $0 \mathrm{~K}$ and at the separation found experimentally $(\mathrm{p}(5 \times 5))$ there is no intermolecular interaction expected.

Despite this, experimental findings indicate large, well-ordered islands with few degrees of freedom, so we conclude that at room temperature, the molecules oscillate between several

closely spaced local potential energy minima, increasing their interaction range and forcing the more open $\mathrm{p}(5 \times 5)$ structure. The lattice parameter of this structure is $14.4 \AA$. 


\section{ACKNOWLEDGMENTS}

We thank the Royal Society for Research Grant RG130038, UK STFC for Facility Development Grant, 2008, (No: FDPG/080), the Academy of Finland for funding under projects \#277829 and \#218186 and CSC - IT Center for Science for computational resources. We thank A. A. Cafolla for providing the DBBA molecules. We thank D. Hesp and T. Whittles for experimental assistance. We thank T. Mercer for providing a critical reading of the manuscript.

\section{REFERENCES}

[1] X. Li, W. Cai, J. An, S. Kim, J. Nah, D. Yang, R. Piner, A. Velamakanni, I. Jung, E. Tutuc, S. K. Banerjee, L. Colombo, and R. S. Ruoff, Science 324, 1312 (2009).

[2] F. Schwierz, Nat. Nanotechnol. 5, 487 (2010).

[3] O. V. Yazyev, Acc. Chem. Res. 46, 2319 (2013).

[4] M. Sarkar and A. Samanta, Acta Cryst. E 59, o1764 (2003).

[5] J. Cai, P. Ruffieux, R. Jaafar, M. Bieri, T. Braun, S. Blankenburg, M. Muoth, A. P. Seitsonen, M. Saleh, X. Feng, K. Müllen, and R. Fasel, Nature 466, 470 (2010).

[6] Q. Fan, J. M. Gottfried, and J. Zhu, Acc. Chem. Res. 48, 2484 (2015).

[7] M. Kolmer, Z. A. A. Ahmad, J. S. Prauzner-Bechcicki, W. Piskorz, F. Zasada, S. Godlewski, B. Such, Z. Sojka, and M. Szymonski, Angew. Chem. Int. Ed. 125, 10490 (2013).

[8] H. Huang, D. Wei, J. Sun, S. L. Wong, Y. P. Feng, A. H. C. Neto, and A. T. S. Wee, Sci. Rep. 2, 983 (2012).

[9] K. A. Simonov, N. A. Vinogradov, A. S. Vinogradov, A. V. Generalov, E. M. Zagrebina, N. Maårtensson, A. A. Cafolla, T. Carpy, J. P. Cunniffe, and A. B. Preobrajenski, J. Phys. Chem. C 118, 12532 (2014).

[10] C. Sánchez-Sánchez, T. Dienel, O. Deniz, P. Ruffieux, R. Berger, X. Feng, K. Müllen, and R. Fasel, ACS Nano 10, 8006 (2016). 
[11] K. A. Simonov, N. A. Vinogradov, A. S. Vinogradov, A. V. Generalov, E. M. Zagrebina, G. I. Svirskiy, A. A. Cafolla, T. Carpy, J. P. Cunniffe, T. Taketsugu, A. Lyalin, N. Mårtensson, and A. Preobrajenski, ACS Nano 9, 8997 (2015).

[12] P. Y. Huang, C. S. Ruiz-Vargas, A. M. van der Zande, W. S. Whitney, M. P. Levendorf, J. W. Kevek, S. Garg, J. S. Alden, C. J. Hustedt, Y. Zhu, J. Park, P. L. McEuen, and D. A. Muller, Nature 469, 389 (2011).

[13] H. I. Rasool, E. B. Song, M. Mecklenburg, B. C. Regan, K. L. Wang, B. H. Weiller, and J. K. Gimzewski, J. Am. Chem. Soc. 133, 12536 (2011).

[14] A. W. Tsen, L. Brown, M. P. Levendorf, F. Ghahari, P. Y. Huang, R. W. Havener, C. S. Ruiz-Vargas, D. A. Muller, P. Kim, and J. Park, Science 336, 1143 (2012).

[15] Z. Fei, A. S. Rodin, W. Gannett, S. Dai, W. Regan, M. Wagner, M. K. Liu, A. S. McLeod, G. Dominguez, M. Thiemens, A. H. C. Neto, F. Keilmann, A. Zettl, R. Hillenbrand, F. M. M., and D. N. Basov, Nat. Nanotechnol. 8, 821 (2013).

[16] P. Vancsó, G. I. Márk, P. Lambin, A. Mayer, Y. Kim, C. Hwang, and L. P. Biró, Carbon 64, $101(2013)$.

[17] S. M. Barlow and R. Raval, Surf. Sci. Rep. 50, 201 (2003).

[18] M. Batzill, Surf. Sci. Rep. 67, 83 (2012).

[19] P. Han, K. Akagi, F. F. Canova, H. Mutoh, S. Shiraki, K. Iwaya, P. S. Weiss, N. Asao, and T. Hitosugi, ACS Nano 8, 9181 (2014).

[20] G. Kresse and J. Hafner, Phys. Rev. B 47, 558 (1993).

[21] G. Kresse and J. Hafner, J. Phys.: Condens. Matter 6, 8245 (1994).

[22] G. Kresse and J. Hafner, Phys. Rev. B 49, 14251 (1994).

[23] G. Kresse and J. Furthmüller, Comput. Mater. Sci. 6, 15 (1996).

[24] P. E. Blöchl, Phys. Rev. B 50, 17953 (1994).

[25] J. P. Perdew, K. Burke, and M. Ernzerhof, Phys. Rev. Lett. 77, 3865 (1996).

[26] H. J. Monkhorst and J. D. Pack, Phys. Rev. B 13, 5188 (1976).

[27] J. Klimeš, D. R. Bowler, and A. Michaelides, Phys. Rev. B 83, 195131 (2011).

[28] R. Cortés, A. Mascaraque, P. Schmidt-Weber, H. Dil, T. U. Kampen, and K. Horn, Nano Lett. 8, 4162 (2008).

[29] T. Carpy, A scanning tunnelling microscopy and spectroscopic study of bromine functionalised molecules on metal surfaces, Ph.D. thesis, Dublin City University (2015). 